\title{
Isolation of ${ }^{35} \mathrm{~S}-$ and ${ }^{3} \mathrm{H}$-Labelled Proteoglycans from Cultures of Human Embryonic Skin Fibroblasts
}

\author{
By Lars CÖSTER, Ingemar CARLSTEDT and Anders MALMSTRÖM \\ Department of Physiological Chemistry 2, University of Lund, P.O. Box 750, S-220 07 Lund, Sweden
}

(Received 23 April 1979)

\begin{abstract}
${ }^{35} \mathrm{SO}_{4}{ }^{2-}$ - and $\left[{ }^{3} \mathrm{H}\right]$-leucine-labelled proteoglycans were isolated from the medium of a fibroblast culture, from an EDTA extract of the monolayer, and from consecutive dithiothreitol and guanidine hydrochloride extracts of the cells. Proteoglycans of different sizes were isolated from the extracts by gel chromatography on Sepharose 4B. In the medium and the EDTA extract the largest proteoglycans contained only ${ }^{35} \mathrm{~S}$-labelled galactosaminoglycan, whereas all other fractions contained in addition heparan $\left[{ }^{35} \mathrm{~S}\right] \mathrm{sul}-$ phate. The galactosaminoglycan-containing proteoglycans of the various extracts were separated into a larger component, containing chondroitin sulphate-like side chains, and a smaller component, containing dermatan sulphate. The larger proteoglycan of the medium showed reversible association-dissociation behaviour when chromatographed on Sepharose CL2B in phosphate-buffered saline and in 4M-guanidine hydrochloride respectively. This property remained after removal of extraneous proteins by $\mathrm{CsCl}$-densitygradient centrifugation in guanidine hydrochloride. The association was markedly increased by the addition of high-molecular-weight hyaluronic acid.
\end{abstract}

Fibroblasts in culture have been shown to synthesize and secrete glycosaminoglycans (Grossfeld et al., 1957; Matalon \& Dorfman, 1966; Saito \& Uzman, 1971 ), and by now a vast literature has accumulated on this subject. It has been reported that fibroblasts from different tissues of the same species synthesize different amounts of the various glycosaminoglycans (Conrad et al., 1977) and galactosaminoglycans with different structure (Sjöberg \& Fransson, 1977). Cultured cells distribute newly synthesized proteoglycans to the medium, to a cell-associated pool and some is retained intracellularly (Fratantoni et al., 1968; Kresse et al., 1975). The glycosaminoglycan composition varies between the different pools in cultures of skin fibroblasts. Hyaluronic acid constitutes the major polysaccharide of the medium (Matalon \& Dorfman, 1966), whereas heparan sulphate is a characteristic component of the cellassociated material (Kraemer, 1971). Both the relative

Abbreviations used: PG-M, proteoglycans obtained from the medium; PG-EDTA, proteoglycans obtained from the EDTA extract; PG-DTT, proteoglycans obtained from the dithiothreitol extract; PG-CD, proteoglycans from the guanidine hydrochloride extract of cells recovered after the dithiothreitol extraction; PG-C, proteoglycans obtained from a guanidine hydrochloride extract of the cells recovered after the EDTA extraction; PG-F, proteoglycans obtained from a guanidine hydrochloride extract of the substratum. distribution of glycosaminoglycans and the structure of the polysaccharides vary between the different pools. Thus dermatan sulphate of the cell-associated material is rather glucuronic acid-rich, whereas intracellular dermatan sulphate is more iduronic acid-rich (Malmström et al., 1975).

In contrast with the abundance of data on glycosaminoglycans, little is known about their organization at the proteoglycan level. Kresse et al. (1975) reported the presence of proteoglycans of different size in the culture medium and in an EDTA extract of the monolayer. No further characterization was performed. It was therefore decided to isolate proteoglycans from fibroblast cultures and to study the structure and arrangement of polysaccharide chains on the polypeptide core.

In this report a procedure for the isolation of proteoglycans from the various compartments of a fibroblast culture is described. The proteoglycans were separated into subfractions with different proportions of heparan sulphate and dermatan sulphate and with distinctly different structures for the galactosaminoglycan component. Proteoglycans from the medium (a major part of the total) were isolated by methods that avoid dissociative and denaturing agents. A glucuronic acid-rich subfraction of these proteoglycans displayed reversible aggregation. A preliminary report of this work has appeared (Cöster et al., 1977). 


\section{Experimental}

\section{Materials}

Papain (twice crystallized) and guanidine hydrochloride (technical grade) were purchased from Sigma Chemical Co., St. Louis, MO, U.S.A. Stock solutions of $7 \mathrm{M}$-guanidine hydrochloride were left overnight, with stirring, with activated charcoal, and subsequently filtered through filter paper. After this procedure the $A_{280}$, read against water, was less than 0.1. Chondroitinase AC (EC 4.2.2.5) was manufactured by Seikagaku Kogyo Co., Tokyo, Japan, and dithiothreitol was obtained from Miles Laboratories, Kankakee, IL, U.S.A. ${ }^{35} \mathrm{SO}_{4}{ }^{2-}$ (carrier-free) and $\mathrm{L}-\left[4,5-{ }^{3} \mathrm{H}\right]$ leucine $(40-60 \mathrm{Ci} / \mathrm{mmol})$ were products of The Radiochemical Centre, Amersham, Bucks., U.K. Materials used for cell cultures (Earle's minimal essential medium, calf serum and antibiotics) were obtained from sources listed previously (Malmström et al., 1975). Insta-Gel was bought from Packard Instrument AB, Bandhagen, Sweden. Sepharose and Sephadex gels were obtained from Pharmacia Fine Chemicals, Uppsala, Sweden, and microgranular ion-exchange DEAE-cellulose (DE32) was from Whatman. Ultrafiltration membranes (XM-100 and PM-10) were manufactured by Amicon.

Carrier proteoglycan [fraction A1D1D1 which is free from hyaluronic acid (Heinegård, 1972)] was prepared from bovine nasal cartilage. Fraction A1D1D1 was subsequently reduced and alkylated (Heinegård, 1977) and designated A1D1D1-RA.

\section{Analytical methods}

Radioactivity was determined in a Packard 2450 scintillation counter. For the determination of ${ }^{35} \mathrm{SO}_{4}{ }^{2-}$ alone, $2.0 \mathrm{ml}$ of sample and $3.0 \mathrm{ml}$ of InstaGel were used. For simultaneous ${ }^{35} \mathrm{SO}_{4}{ }^{2-}$ and ${ }^{3} \mathrm{H}$ determination $0.5 \mathrm{ml}$ and $5 \mathrm{ml}$ were used respectively. Counting efficiency was $70 \%$ when ${ }^{35} \mathrm{SO}_{4}{ }^{2-}$ was determined alone and $60 \%\left({ }^{35} \mathrm{SO}_{4}{ }^{2-}\right)$ and $24 \%\left({ }^{3} \mathrm{H}\right)$ when ${ }^{35} \mathrm{SO}_{4}{ }^{2-}$ and ${ }^{3} \mathrm{H}$ were determined together.

Analytical gel chromatography was performed as described in the legends to the Figures. Carrier proteoglycan or polysaccharide $(1 \mathrm{mg} / \mathrm{ml})$ was added if not otherwise stated. In order to remove possible small amounts of insoluble material before application to the columns the samples were centrifuged at $30000 \mathrm{~g}$ for $20 \mathrm{~min}$. The recovery of labelled material after chromatography was generally between 70 and $90 \%$.

\section{Density-gradient centrifugation}

$\mathrm{CsCl}$ was added to a proteoglycan solution that contained $4 \mathrm{M}$-guanidine hydrochloride $/ 0.05 \mathrm{M}$ sodium acetate, pH 5.8, until a starting density of $1.35 \mathrm{~g} / \mathrm{ml}$ was obtained. Centrifugation was performed in an MSE SuperSpeed 65 centrifuge with an
$8 \times 25 \mathrm{ml}(16 \mathrm{ml}$ of solution/tube $)$ angle rotor at $10^{\circ} \mathrm{C}$ and $34000 \mathrm{rev} . / \mathrm{min}$. for $48 \mathrm{~h}$. Tubes were emptied into $1 \mathrm{ml}$ fractions with the aid of an MSE tube piercer. The density of the fractions was determined with a $200 \mu \mathrm{l}$ micropipette as pycnometer. Radioactivity was determined on portions of the fractions. The fractions were pooled and stored frozen in $4 \mathrm{M}$-guanidine hydrochloride. Samples of each of the eight pooled bottom fractions were subjected to a second centrifugation in 4M-guanidine hydrochloride/0.05 $\mathrm{M}$-sodium acetate, $\mathrm{pH} 5.8$, in $\mathrm{CsCl}$ with a starting density of $1.51 \mathrm{~g} / \mathrm{ml}$. The conditions were otherwise as above.

\section{Degradative procedures}

$\mathrm{HNO}_{2}$ treatment. The ${ }^{35} \mathrm{~S}$-labelled glycosaminoglycans (containing $0.5 \mathrm{mg}$ of heparan sulphate and $0.5 \mathrm{mg}$ of dermatan sulphate as carriers) were dissolved in $1 \mathrm{ml}$ of $0.24 \mathrm{M}-\mathrm{NaNO}_{2} / 1.8 \mathrm{M}$-acetic acid and kept at room temperature for $80 \mathrm{~min}$ (Lagunoff $\&$ Warren, 1962). The reaction was terminated by repeated evaporation of $\mathrm{HNO}_{2}$ after the addition of a few drops of methanol. $\mathrm{HNO}_{2}$ cleaves heparan sulphate into small oligosaccharide fragments, which were separated from the galactosaminoglycans by gel chromatography.

Periodate oxidation. ${ }^{35} \mathrm{~S}$-labelled galactosaminoglycans were oxidized with $0.02 \mathrm{M}-\mathrm{NaIO}_{4}$ in $0.05 \mathrm{M}$ sodium citrate or formate, $\mathrm{pH} 3.0$. for $24 \mathrm{~h}$ at $4^{\circ} \mathrm{C}$, and subsequently cleaved by alkaline elimination at pH 12 for $30 \mathrm{~min}$ at room temperature (Fransson, 1974). This method cleaves unsubstituted iduronic acid residues in the galactosaminoglycans (see Table 1). Subsequent gel chromatography permits quantification of non-sulphated iduronic acid residues (for details see Table 1), as well as suggesting the distribution along the chain of these units.

Digestion with chondroitinase $A C .{ }^{35}$ S-labelled galactosaminoglycans were dissolved in $0.2 \mathrm{M}$-Tris/ $\mathrm{HCl} / 0.2 \mathrm{M}$-sodium acetate buffer, $\mathrm{pH} 8.0$, containing 0.02 unit of enzyme, $0.1 \mathrm{mg}$ of bovine serum albumin and $0.5 \mathrm{mg}$ of carrier chondroitin sulphate (Suzuki et al., 1968). After $12 \mathrm{~h}$ of incubation at $37^{\circ} \mathrm{C}$ the digest was chromatographed on a Sephadex G-50 column.

Incorporation of ${ }^{35} \mathrm{SO}_{4}{ }^{2-}$ and $\left[{ }^{3} \mathrm{H}\right]$ leucine into proteoglycans by cultured fibroblasts

Fibroblast cultures were established from human embryonic skin and were grown in monolayers as described by Malmström et al. (1975). Before addition of radioactive precursors, the medium was changed to a sulphate-poor medium (minimum essential medium with Earle's salts in which the sulphate concentration was decreased to $0.11 \mathrm{~mm}$ ). After 3 days this medium was removed and the labelling medium, which contained $5 \mu \mathrm{Ci}$ of carrier-free 
Culture medium

Concentration by ultrafiltration

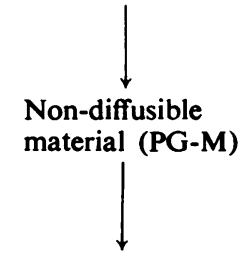

Gel chromatography on Sepharose 4B

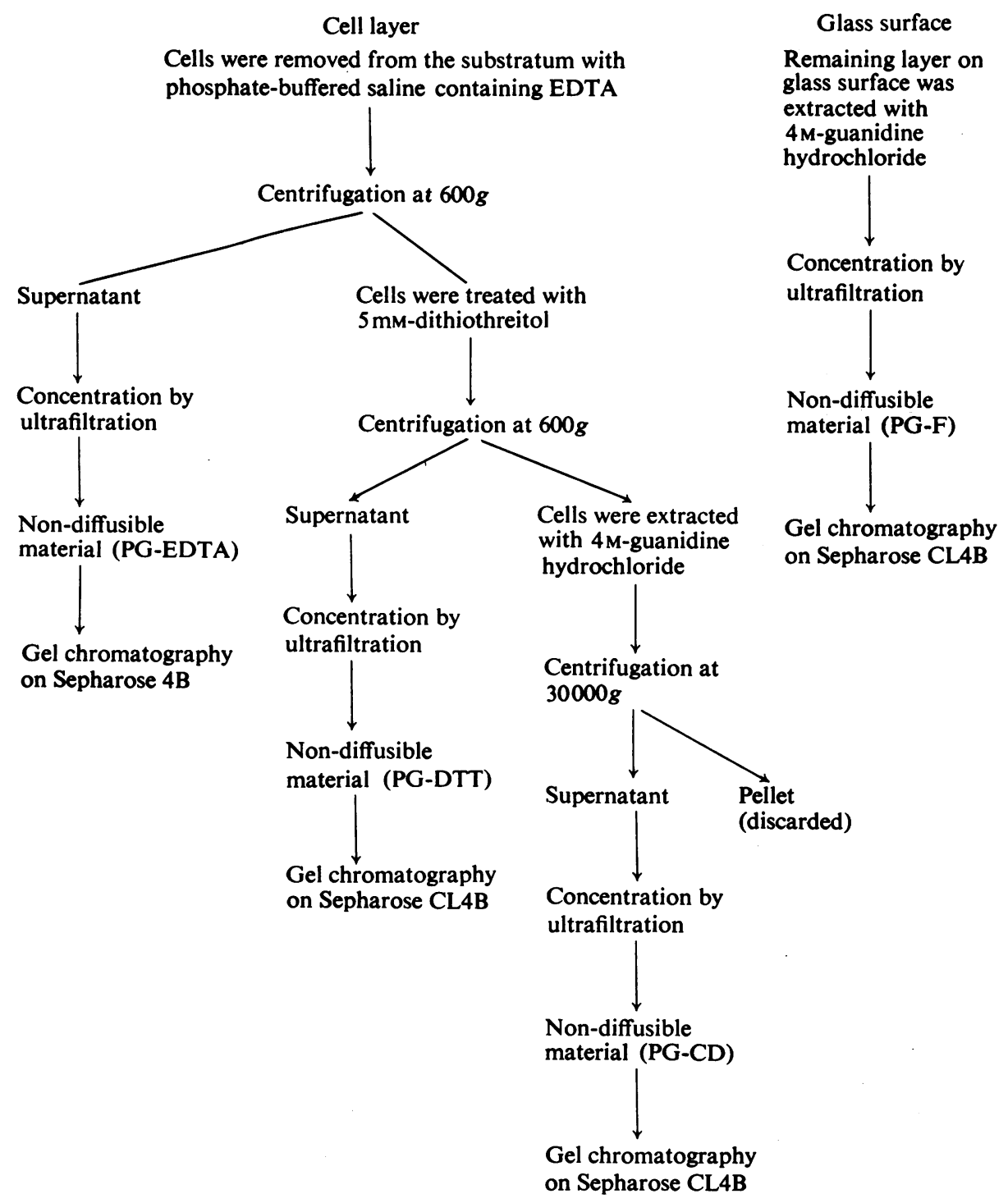

Scheme 1. Flow scheme for isolation of proteoglycans
${ }^{35} \mathrm{SO}_{4}{ }^{2-}$ and $\left[{ }^{3} \mathrm{H}\right]$ leucine/ml, was added. The final concentrations of sulphate and leucine were 0.11 and $0.004 \mathrm{~mm}$ respectively. The cells were incubated with the radioactive precursors for $72 \mathrm{~h}$.

\section{Isolation of proteoglycans}

The culture medium was decanted and the cell layer was carefully washed with $10 \mathrm{ml}$ of phosphatebuffered saline $(137 \mathrm{~mm}-\mathrm{NaCl} / 3 \mathrm{mM}-\mathrm{KCl} / 8 \mathrm{~mm}-$ $\mathrm{Na}_{2} \mathrm{HPO}_{4} / 1 \mathrm{~mm}-\mathrm{KH}_{2} \mathrm{PO}_{4}$, pH 7.4). The medium and the washings were combined, centrifuged at
$10000 \mathrm{~g}$ for $30 \mathrm{~min}$, and the resulting supernatant was supplemented with $0.1 \mathrm{M}$-di-isopropyl phosphorofluoridate in propanol to a final concentration of $0.001 \mathrm{M}$. This fraction (PG-M) was diluted $(1: 1)$ with phosphate-buffered saline containing $0.2 \mathrm{M}$-6-aminohexanoic acid, 0.02M-EDTA disodium, $0.01 \mathrm{M}$ benzamidinium chloride and cartilage proteoglycan $(5 \mathrm{mg} / 100 \mathrm{ml}$ of fraction A1D1D1-RA) as carrier. The medium was concentrated 10 times in a Diaflo ultrafiltration cell (XM-100 membrane) at $4^{\circ} \mathrm{C}$, whereafter it was dialysed overnight against phos- 


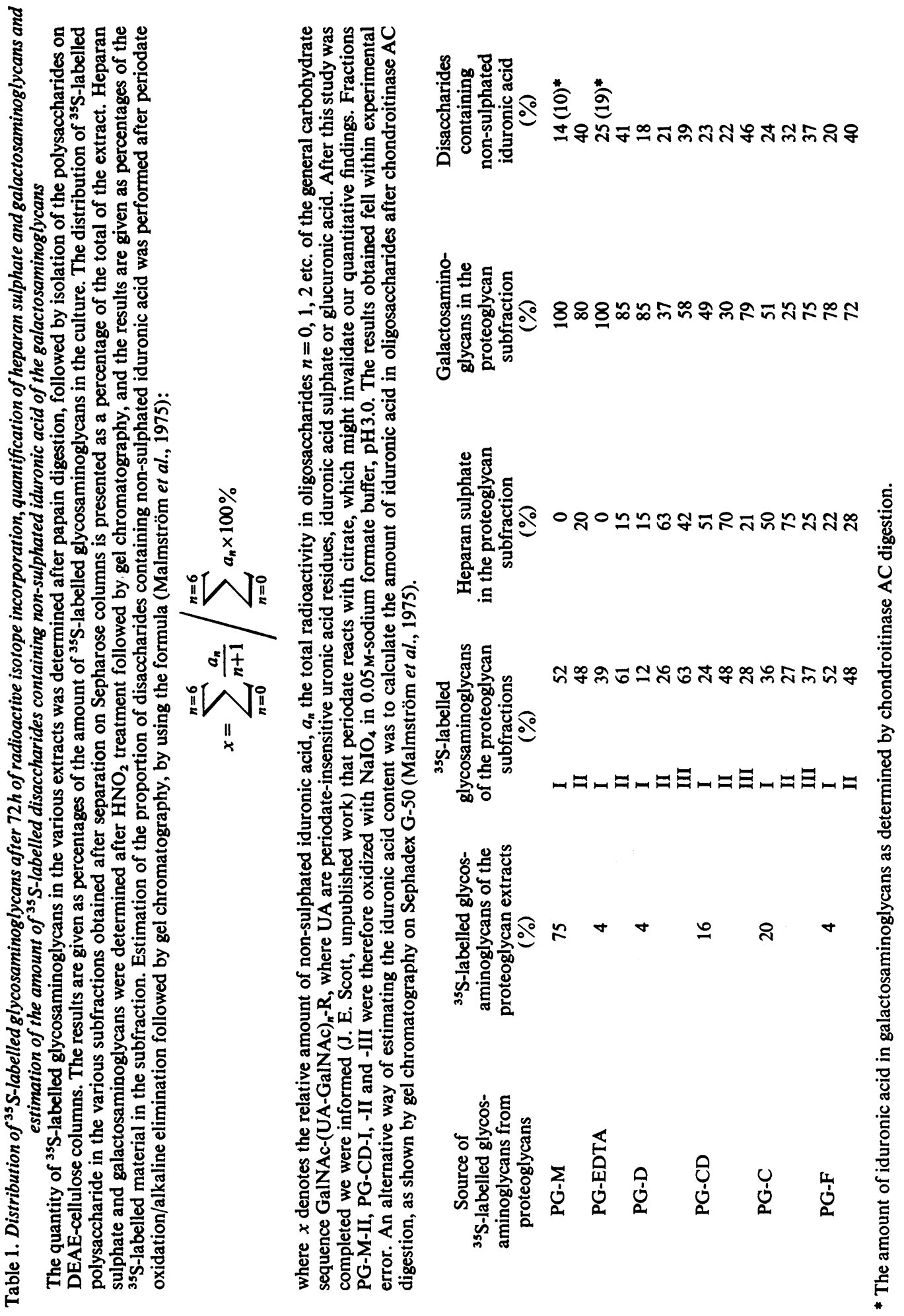


phate-buffered saline, $\mathrm{pH} 7.4$, containing the proteolytic inhibitors $0.1 \mathrm{M}$-6-aminohexanoic acid, $0.01 \mathrm{M}$ EDTA and $0.005 \mathrm{M}$-benzamidinium chloride. The non-diffusible material was chromatographed on Sepharose 4B. The diffusible material from the ultrafiltration step was concentrated in a Diaflo cell over a PM-10 membrane at $4^{\circ} \mathrm{C}$.

The cell layer was treated with $0.5 \mathrm{~mm}$-EDTA in phosphate-buffered saline in order to detach the cells from the glass surface. The substratum was carefully washed twice with $10 \mathrm{ml}$ of the same buffer. The cell suspension and the washings were combined and centrifuged at $600 \mathrm{~g}$ for $10 \mathrm{~min}$. The supernatant (PG-EDTA fraction) was treated as was the PG-M fraction above, except that instead of a XM-100 membrane a PM-10 membrane was used during the ultrafiltration. The remaining cells were treated with $0.005 \mathrm{M}$-dithiothreitol in phosphate-buffered saline, $\mathrm{pH} 7.4$, for $1 \mathrm{~h}$ at $37^{\circ} \mathrm{C}$, with gentle shaking. The cell suspension was centrifuged at $600 \mathrm{~g}$ for $10 \mathrm{~min}$. The supernatant (PG-DTT fraction) was treated with iodoacetic acid (final concentration of $0.015 \mathrm{M}$ ), left for $2 \mathrm{~h}$ at $4^{\circ} \mathrm{C}$ and then concentrated by ultrafiltration through a PM-10 membrane. The remaining cells were extracted twice for $12 \mathrm{~h}$ with $4 \mathrm{M}$-guanidine hydrochloride containing the above-mentioned proteolytic inhibitors and $0.015 \mathrm{M}$-iodoacetic acid, pH7.4. In an alternative procedure, cells that had been treated with EDTA were directly extracted with $4 \mathrm{M}$-guanidine hydrochloride $/ 0.005 \mathrm{M}$-Tris $/ \mathrm{HCl}$, pH 7.4, containing proteolytic inhibitors. The guanidine hydrochloride extract of the cells was cleared of cell debris by centriguation at $30000 \mathrm{~g}$ for $30 \mathrm{~min}$, and the supernatant (PG-CD or PG-C fraction) was concentrated by ultrafiltration through a PM-10 membrane.

The material remaining on the flask surface after detachment of the cells was extracted with $4 \mathrm{M}$ guanidine hydrochloride ( $35 \mathrm{ml} /$ flask) containing the same inhibitors as mentioned above and treated as was the PG-CD fraction. This fraction was designated PG-F. A flow chart summarizing the isolation procedure is shown in Scheme 1.

To ensure that no significant amount of material was lost during the isolation procedure, samples were taken from the various fractions before and after the ultrafiltration steps and the recovery of ${ }^{35}$ S-labelled polysaccharide material was determined as described below. Furthermore, the content of ${ }^{35}$ S-labelled polysaccharide was determined in the remaining cell debris after guanidine hydrochloride extraction.

\section{Isolation and identification of glycosaminoglycans}

The radioactively labelled samples from various fractions (containing $0.5 \mathrm{mg}$ of dermatan sulphate and $0.5 \mathrm{mg}$ of heparan sulphate $/ \mathrm{ml}$ as carrier) were digested with papain (three additions of $0.6 \mathrm{mg} / \mathrm{ml}$ ) in $1.0 \mathrm{M}-\mathrm{NaCl} / 0.010 \mathrm{M}-\mathrm{EDTA}_{2} / 0.010 \mathrm{M}$-cysteine hydrochloride $/ 0.050 \mathrm{M}$-sodium phosphate buffer, pH7.0, for $24 \mathrm{~h}$ at $65^{\circ} \mathrm{C}$. The digests were dialysed for 3 days against several changes of water and were brought to $0.2 \mathrm{M}$-acetic acid/pyridine buffer, $\mathrm{pH} 5.0$, by the addition of concentrated acetic acid/pyridine buffer, pH5.0. The samples were chromatographed on DEAE-cellulose columns $(3 \mathrm{~mm} \times 60 \mathrm{~mm})$ equilibrated with $0.2 \mathrm{M}$-acetic acid/pyridine buffer, $\mathrm{pH}$ 5.0. The columns were eluted stepwise with $2 \mathrm{ml}$ of each of $0.2 \mathrm{M}-, 0.8 \mathrm{M}-, 0.9 \mathrm{M}$ - and $3.0 \mathrm{M}$-acetic acid/pyridine buffer, pH 5.0. The first three steps removed small amounts of non-glycosaminoglycan ${ }^{35} \mathrm{SO}_{4}{ }^{2-}$ activity. The ${ }^{35}$ S-labelled polysaccharides were then recovered in the $3.0 \mathrm{M}$ fraction (Sjöberg et al., 1979). The polysaccharide fractions were treated with $\mathrm{HNO}_{2}$ and subjected to gel chromatography to quantify heparan sulphate. The resistant galactosaminoglycans were recovered from the void volume of the column and subjected to periodate oxidation/ alkaline elimination followed by gel chromatography to assess the distribution of iduronic acid in the polymers.

\section{Results}

Yield and subfractionation of proteoglycans from the various compartments

After $72 \mathrm{~h}$ of radioactive isotope incorporation most $(75 \%)$ of the ${ }^{35}$ S-labelled proteoglycans was recovered from the medium (Table 1). Recovery of ${ }^{35}$ S-labelled polysaccharide in the non-diffusible material after ultrafiltration and dialysis on an XM-100 filter was $90-95 \%$, which indicated that all ${ }^{35}$ S-containing material was of high molecular weight. The guanidine hydrochloride extract of the cell accounted for $16 \%$ of the proteoglycans. Less than $1 \%$ was recovered in the residue obtained after centrifugation of the extract. Small amounts of proteoglycans were obtained in the EDTA extract (PG-EDTA fraction, 4\%), the dithiothreitol extract of the cells (PG-DTT fraction, $4 \%$ ) and the guanidine hydrochloride extract of the substratum (PG-F fraction, $1 \%$ ). When the dithiothreitol extraction step was omitted, $20 \%$ of the total ${ }^{35}$ S-labelled polysaccharide was found in the guanidine hydrochloride extract of the cells.

After concentration by ultrafiltration and removal of low-molecular-weight substances by dialysis the various fractions were chromatographed on Sepharose 4B (Figs. 1 and 2). Each fraction could be separated into at least one excluded and one or two included polydisperse proteoglycan populations. Fractions were pooled as indicated in the Figures, and the relative yields of the isolated components are summarized in Table 1. As shown in Fig. 1, the 


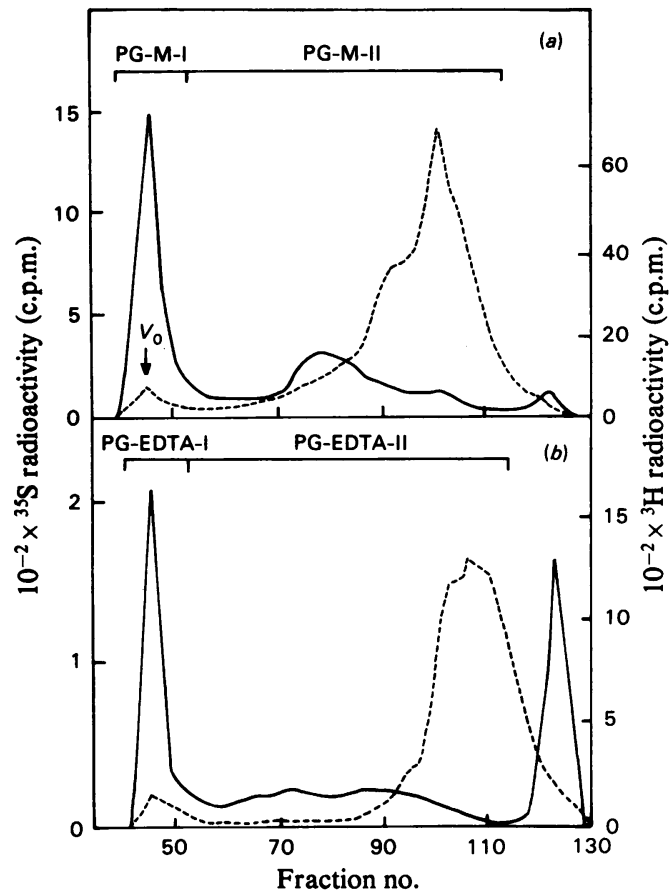

Fig. 1. Gel chromatography on Sepharose $4 B$ of the medium (a) and the EDTA fraction (b)

Radioactive proteoglycans were isolated as described in the Experimental section and subjected to gel chromatography on Sepharose 4B. Column size was $4 \mathrm{~cm} \times 210 \mathrm{~cm}$, and the eluent phosphate-buffered saline, pH 7.4, in (a) and phosphate-buffered saline containing $10 \mathrm{~mm}$-EDTA in $(b)$; elution rate was $45 \mathrm{ml} / \mathrm{h}$, and the fraction size $25 \mathrm{ml} ; V_{0}$, void volume of the column; _- , ${ }^{34} \mathrm{SO}_{4}$ (c.p.m.); - ---, ${ }^{3} \mathrm{H}$ (c.p.m.). The medium was concentrated to $50 \mathrm{ml}$ and the EDTA extract to $30 \mathrm{ml}$. The medium fraction was then dialysed against phosphate-buffered saline. Both fractions were subsequently centrifuged to remove precipitated protein and applied to the column. Less than $1 \%$ of the total ${ }^{35} \mathrm{~S}$-labelled material was removed from the precipitate. The proteoglycan subfractions were pooled as indicated by the bars.

proteoglycans of the medium and the EDTA extract were similar and contained a large amount of ${ }^{35} \mathrm{~S}$ labelled material in the void volume (52 and $39 \%$ respectively) and a spectrum of proteoglycans of different molecular size included on the gel. The guanidine hydrochloride extract of the cells contained less of the excluded and more of the included species (Fig. 2b). The latter could be resolved into two different subfractions, the larger of which was the quantitatively dominating one $(48 \%)$. The proteoglycan fraction of the dithiothreitol extract contained mainly small proteoglycans, although significant amounts of proteoglycans of intermediary and large sizes were present. The material extracted from

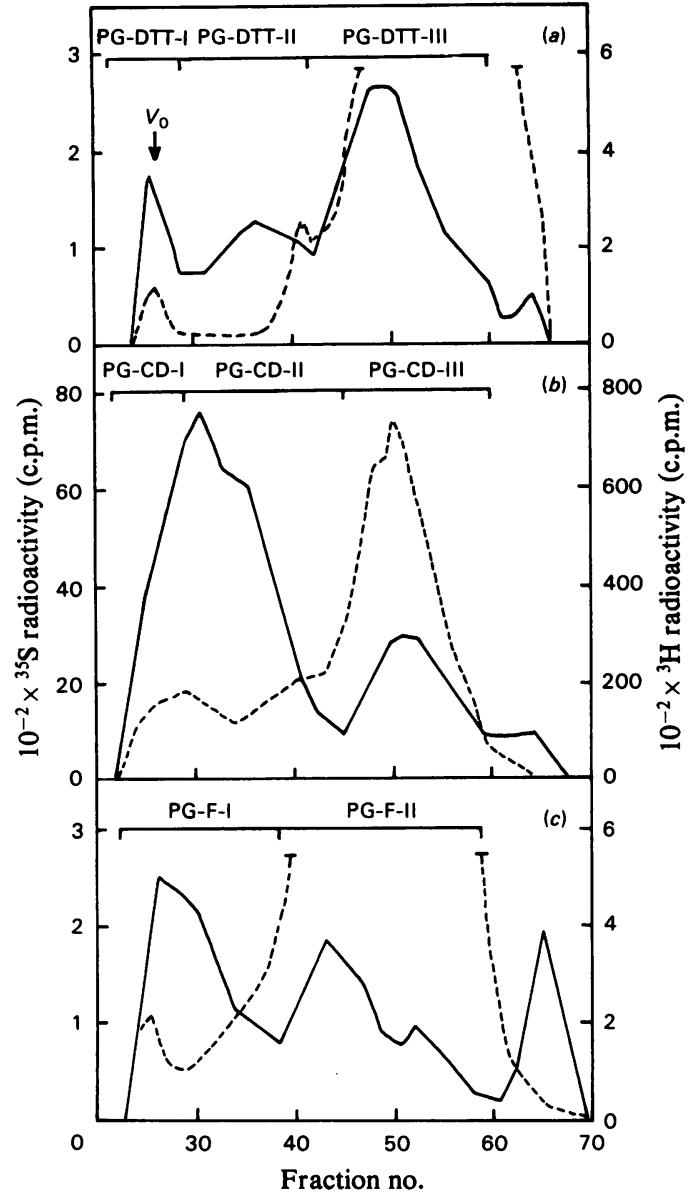

Fig. 2. Gel chromatography on Sepharose CL4B of the dithiothreitol extract (a), the guanidine hydrochloride extract of the cells $(b)$ and the guanidine hydrochloride extract of the substratum (c)

Radioactive proteoglycans were isolated as described in the Experimental section and subjected to gel chromatography on Sepharose CL4B. Column size was $1.4 \mathrm{~cm} \times 140 \mathrm{~cm}$, and the eluent phosphatebuffered saline, $\mathrm{pH} 7.4$, in $(a)$ and $4 \mathrm{M}$-guanidine hydrochloride in $0.005 \mathrm{M}-\mathrm{Tris} / \mathrm{HCl}, \mathrm{pH} 7.0$ in $(b)$ and (c); elution rate was $6 \mathrm{ml} / \mathrm{h}$, and fraction size $3 \mathrm{ml}$; $V_{0}$, void volume of the column;,$-{ }^{35} \mathrm{SO}_{4}$ (c.p.m.); - - , ${ }^{3} \mathrm{H}$ (c.p.m.). The fractions were concentrated to $10 \mathrm{ml}$, centrifuged to remove small amounts of insoluble protein and applied to the column. Less than $1 \%$ of ${ }^{35} \mathrm{SO}_{4}$-labelled material could be recovered from the precipitate. The subfractions were pooled as shown by the bars. Attempts to chromatograph PG-CD, PG-C and PG-F in phosphate-buffered saline were unsuccessful because of precipitation of the material.

the glass surface of the culture vessel had a chromatographic profile intermediate between the guanidine 
hydrochloride extract and the dithiothreitol extract of the cells. No significant differences could be detected by gel chromatography of the guanidine hydrochloride extract of the cells when the dithiothreitol extraction was omitted.

Distribution of heparan $\left[{ }^{35} S\right]$ sulphate and ${ }^{35} S$-labelled galactosaminoglycans between the various proteoglycan subfractions

Quantification of heparan sulphate and galactosaminoglycans is based on ${ }^{35} \mathrm{~S}$ determination; specific radioactivities were not measured. The amount of heparan sulphate in the various subfractions (Table 1) was determined after $\mathrm{HNO}_{2}$ treatment of ${ }^{35} \mathrm{~S}$-labelled material, followed by separation of the cleaved products on Sephadex G-50. The larger proteoglycan species of the medium (PG-M-I) and the EDTA fraction (PG-EDTA-I) contained no heparan sulphate. All other fractions contained significant amounts of heparan sulphate and certain subfractions of the guanidine hydrochloride extract, and the dithioreitol extract of the cells (PG-CD-II and PG-DTT-II) consisted mostly of this polysaccharide (70\%).

Further characterization of the galactosaminoglycan component

Glycosaminoglycans were isolated from the proteoglycans by papain digestion and chromatography on DEAE-cellulose as described in the Experimental section. ${ }^{35}$ S-labelled galactosaminoglycans were subsequently isolated from the void

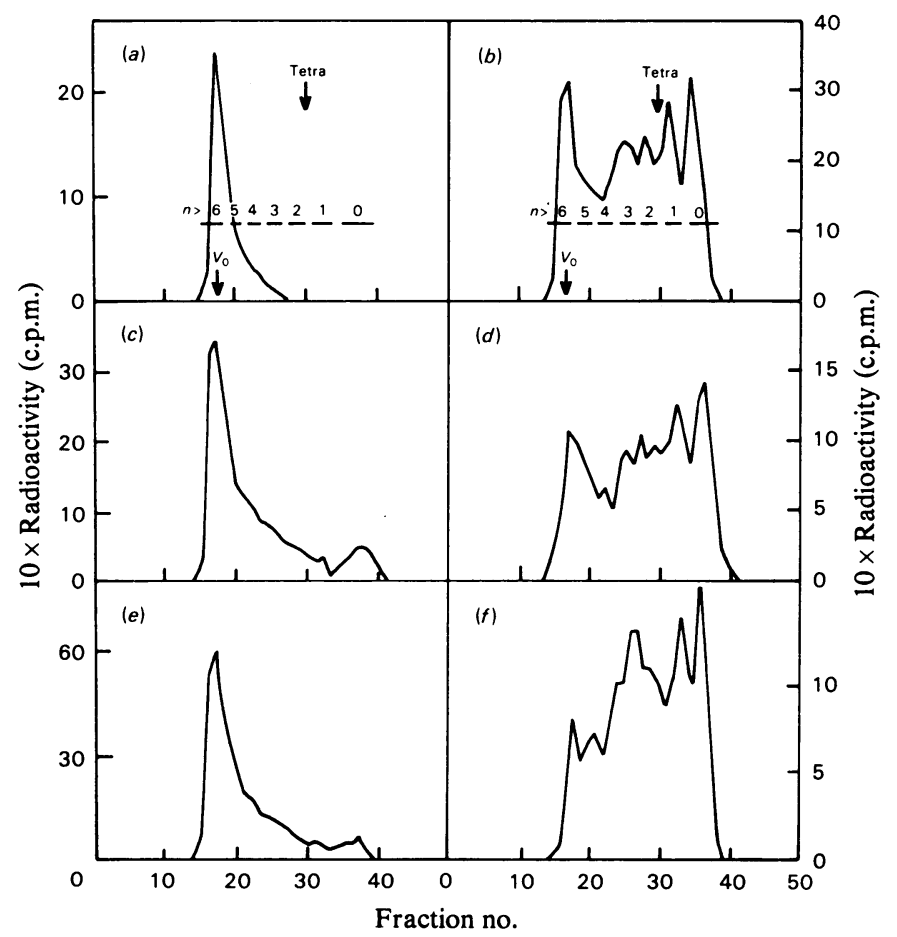

Fig. 3. Gel chromatography on Sephadex $G-50$ of ${ }^{35} S$-labelled galactosaminoglycans after periodate oxidation/alkaline elimination

Polysaccharides were isolated from proteoglycan subfractions by DEAE-cellulose chromatography after papain digestion. The galactosaminoglycan component was obtained after $\mathrm{HNO}_{2}$ treatment by chromatography on Sephadex G-50 and was subsequently subjected to periodate oxidation/alkaline elimination, and the cleaved products were separated by gel chromatography on Sephadex G-50. The ${ }^{35}$ S-labelled material was derived from PG-M-I $(a)$, PG-M-II $(b)$, PG-EDTA-I $(c)$, PD-EDTA-II $(d)$, PG-F-I $(e)$ and PG-F-II $(f)$. Column size was $0.8 \mathrm{~cm} \times 150 \mathrm{~cm}$, and the eluent $0.2 \mathrm{M}$-acetic acid/pyridine buffer, $\mathrm{pH} 5.0$; elution rate was $6 \mathrm{ml} / \mathrm{h} ; V_{0}$, elution volume of Blue Dextran. The elution position of a tetraoligosaccharide standard (from chondroitin sulphate) is indicated by an arrow. To calculate the radioactivity associated with each oligosaccharide fraction the profile was subdivided into subfractions corresponding to oligosaccharides of the general carbohydrate sequence GalNAc-[UA-GalNAc] $n-R$. UA is a periodate-resistant uronic acid, $\mathrm{R}$ is a $\mathrm{C}_{4}$ fragment obtained after periodate oxidation/alkaline elimination (Fransson \& Carlstedt, 1974), and $n=0,1, \ldots 6$. 


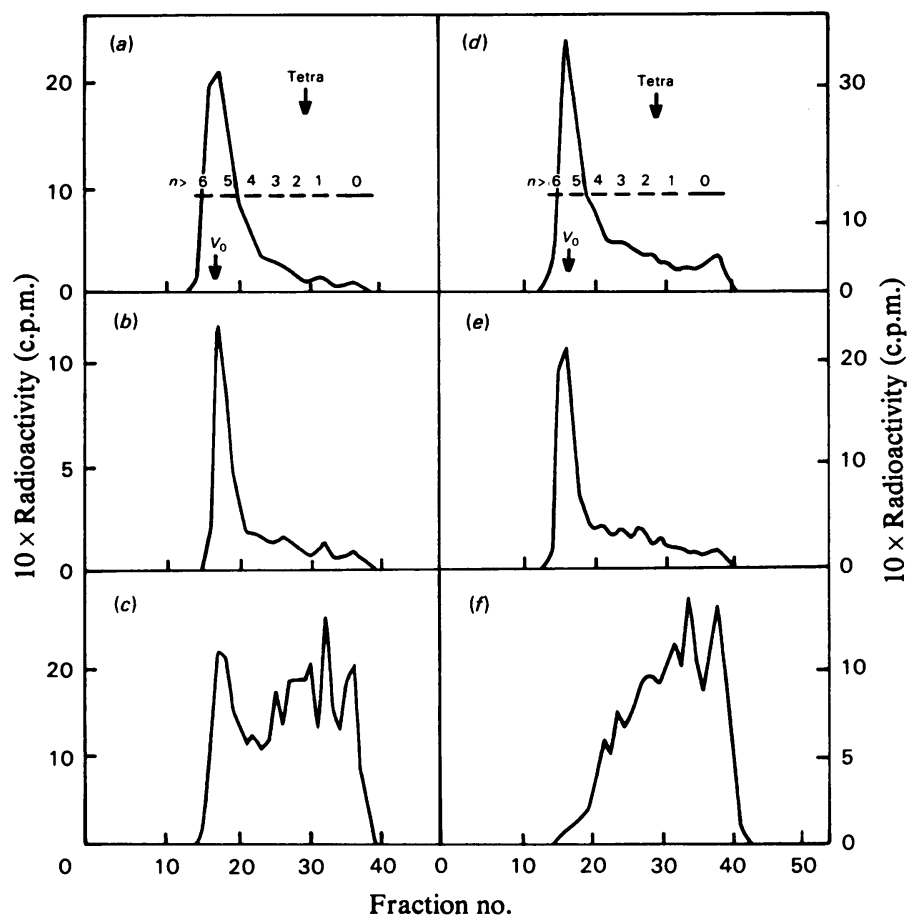

Fig. 4. Gel chromatography on Sephadex G-50 of ${ }^{35}$ S-labelled galactosaminoglycans after periodate oxidation/alkaline elimination

${ }^{35}$ S-labelled material was isolated from PG-DTT-I (a), PG-DTT-II (b), PG-DTT-III (c), PG-CD-I (d), PG-CD-II (e) and PG-CD-III $(f)$ and subjected to periodate oxidation/alkaline elimination followed by chromatography on Sephadex G-50. For further experimental details see Fig. 3 and the Experimental section.

volume of Sephadex G-50 after treatment with $\mathrm{HNO}_{2}$ and subjected to periodate oxidation/alkaline elimination and to chromatography on Sephadex G-50. The results, shown in Figs. 3 and 4, indicate that all fractions contained co-polymers of dermatan sulphate and chondroitin sulphate. There were, however, considerable differences in the uronic acid composition between the various subfractions. Fig. 3 shows that the distribution of non-sulphated iduronic acid in the galactosaminoglycans were similar in the medium (Figs. $3 a$ and $3 b$ ), the EDTA extract (Figs. $3 c$ and $3 d$ ) and the guanidine hydrochloride extract of the substratum (Figs. $3 e$ and $3 f$ ). The polysaccharide portion of the larger proteoglycans of all extracts contained few non-sulphated iduronic acid residues (less than $20 \%$ ), whereas the smaller proteoglycans contained galactosaminoglycans, which were rich in these units (35\% or more) (Figs. 3, 4 and Table 1).

Since nearly all cleaved products, obtained after periodate oxidation and alkaline elimination of the side chains of fraction PG-M-I, were excluded from Sephadex G-50 (Fig. 3a), it is likely that these polysaccharides contained little or no iduronic acid. In order to substantiate this finding polysaccharides were chromatographed on Sepharose CL6B before and after periodate oxidation/alkaline treatment. As shown in Fig. 5, a shift of the elution position of the oxidized material was noted, which indicates that most of the chains contain small amounts of iduronic acid distributed along the polysaccharide chain. This was further confirmed by digestion with chondroitinase AC followed by chromatography of the digest on Sephadex G-50, which showed the presence of small amounts of tetra- and hexa-oligosaccharides (Fig. 6). These results show that the galactosaminoglycans from fraction PG-M-I are co-polymers of chondroitin sulphate and dermatan sulphate, which contain small amounts of iduronic acid-containing disaccharides $(10 \%)$ (Table 1$)$ mostly distributed singly or two together interspaced between long segments of glucuronic acid-containing disaccharides.

Macromolecular properties of proteoglycans from the medium and the EDTA extract

The proteoglycans of the medium and of the EDTA extract were studied further on Sepharose CL2B. Fraction PG-M-I was partially excluded by this gel, while fractions PG-M-II and PG-EDTA-I chroma- 


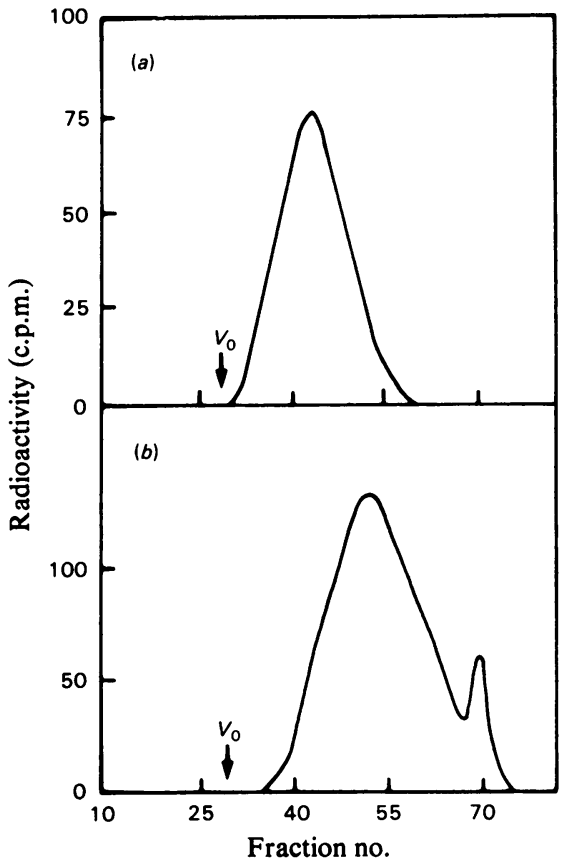

Fig. 5. Gel chromatography of polysaccharide chains from fraction PG-M-I on Sepharose CL6B before (a) and after periodate oxidation/alkaline elimination (b)

Polysaccharide chains were isolated from fraction PG-M-I by DEAE-cellulose chromatography after papain digestion. Before digestion $0.4 \mathrm{mg}$ of carrier dermatan sulphate was added. The free chains were then chromatographed on Sepharose CL6B before $(a)$ and after $(b)$ periodate oxidation/alkaline elimination. Column size was $0.8 \mathrm{~cm} \times 140 \mathrm{~cm}$, and the eluent was $0.5 \mathrm{M}$-guanidine hydrochloride $/ 0.005 \mathrm{M}$ Tris/ $\mathrm{HCl}, \mathrm{pH} 7.0$; elution rate was $3 \mathrm{ml} / \mathrm{h}$; and fraction size $1.1 \mathrm{ml} ; V_{0}$, void volume of the column.

tographed in included positions (Fig. 7). At this stage of the purification the proteoglycans from the medium and the EDTA extract had only been subjected to buffers containing phosphate-buffered saline and in this milieu various interactions can occur. The proteoglycans were therefore subjected to chromatography on Sepharose CL2B in 4M-guanidine hydrochloride. Under these conditions only a slight decrease in molecular size of fraction PG-EDTA-I and no change in molecular size of fractions PG-M-II and PG-EDTA-II was noted, whereas a large change from partially excluded to completely included elution position was recorded for fraction PG-M-I (Fig. 7). This change in hydrodynamic volume for fraction PG-M-I was reversible, as the material resumed its original elution profile when guanidine hydrochloride-treated proteoglycans, after dialysis against phosphate-buffered saline, were rechroma-

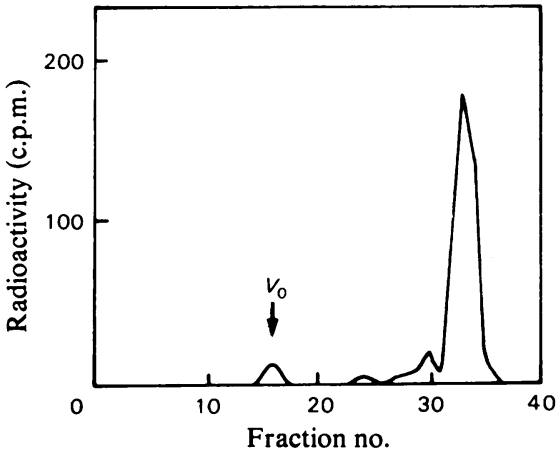

Fig. 6. Gel chromatography of a chondroitinase AC digest of polysaccharide chains from fraction $P G-M-I$ on Sephadex $G-50$

Polysaccharide chains were isolated from fraction PG-M-I by DEAE-cellulose chromatography after papain digestion. Carrier dermatan sulphate $(0.4 \mathrm{mg})$ was added. The sample was subjected to chondroitinase AC digestion, and the digest finally chromatographed on a column of Sephadex G-50. For experimental details see the legend to Fig. 3.

tographed on Sepharose CL2B. In order to gain further information on this reversible associationdissociation phenomenon of fraction PG-M-I, the proteoglycans were subjected to $\mathrm{CsCl}$-densitygradient centrifugation in $4 \mathrm{M}$-guanidine hydrochloride. This step should remove serum proteins of the medium and non-proteoglycan proteins synthesized by the fibroblasts. Most of the ${ }^{35} \mathrm{~S}$-labelled material (Fig. 8a) was recovered from the bottom third of the tube at a density higher than $1.35 \mathrm{~g} / \mathrm{ml}$. After dialysis the proteoglycans were subjected to gel chromatography on Sepharose CL2B in phosphate-buffered saline/4M-guanidine hydrochloride. The chromatographic profiles produced were identical with those before density-gradient centrifugation. Proteoglycans obtained at densities higher than $1.35 \mathrm{~g} / \mathrm{ml}$ were further fractionated, in a gradient with starting density of $1.51 \mathrm{~g} / \mathrm{ml}$, into fractions with different ${ }^{35} \mathrm{~S} /{ }^{3} \mathrm{H}$ ratios from a density of 1.42 to $1.60 \mathrm{~g} / \mathrm{ml}$. The fractions were pooled as indicated in the legend to Fig. $8(b)$, and, after dialysis against phosphate-buffered saline, subjected to gel chromatography on Sepharose CL2B. Most of the proteoglycans, recovered from the bottom fractions, were included on the gel, whereas those equilibrating at lower densities to a larger extent were recovered in the void volume. Since non-proteoglycan proteins are less likely mediators of the aggregation due to the first centrifugation step, other possibilities, such as self-association and/or aggregation induced by components with a buoyant density higher than $1.35 \mathrm{~g} / \mathrm{ml}$, must be considered. Since hyaluronic acid

Vol. 183 


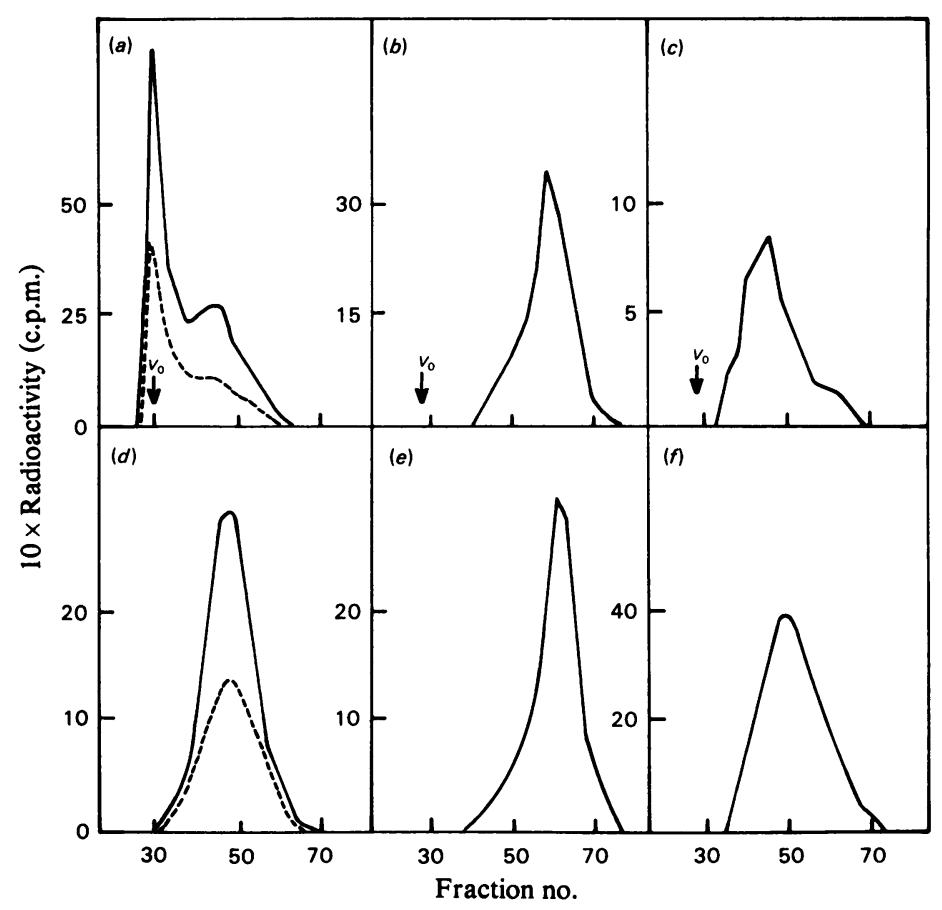

Fig. 7. Gel chromatography on Sepharose CL2B of fractions PG-M-I ( $a$ and d), PG-M-II (b and e) and PG-EDTA-I (c and $f$ ) in phosphate-buffered saline $(a, b$ and $c)$ and $4 M$-guanidine hydrochloride $(d, e$ and $f)$

The proteoglycan fractions were supplemented with $0.25 \mathrm{mg}$ of A1D1D1-RA carrier, dialysed against phosphatebuffered saline or $4 \mathrm{M}$-guanidine hydrochloride $/ 0.005 \mathrm{M}$-Tris/HCl, $\mathrm{pH} 7.0$, and applied to Sepharose CL2B. Column size was $0.6 \mathrm{~cm} \times 140 \mathrm{~cm}$; elution rate was $3 \mathrm{ml} / \mathrm{h}$; fraction size was $0.6 \mathrm{ml}$; eluent was phosphate-buffered saline, $\mathrm{pH} 7.4(a, b$ and $c)$ and $4 \mathrm{M}$-guanidine hydrochloride $/ 0.005 \mathrm{M}-\mathrm{Tris} / \mathrm{HCl}, \mathrm{pH} 7.0(d, e$ and $f) ; V_{0}$, void volume of the column; $\longrightarrow,{ }^{35} \mathrm{SO}_{4}$ (c.p.m.); - ---, ${ }^{3} \mathrm{H}$ (c.p.m.).

is known to induce aggregation of cartilage proteoglycans (Hardingham \& Muir, 1974; Hascall \& Heinegård, 1974), this possibility was considered. High-molecular-weight hyaluronic acid was therefore added to the bottom fractions (density $1.56 \mathrm{~g} / \mathrm{ml}$ ), which should contain small amounts of endogenous hyaluronic acid. However, no alteration in chromatographic properties was induced (Fig. 9). The proteoglycans obtained at lower densities (1.43$1.56 \mathrm{~g} / \mathrm{ml}$ ) and thus containing endogenous hyaluronic acid were, however, more aggregated. Addition of exogenous hyaluronic acid increased the proportion of aggregates from approx. 40 to $75 \%$ of the total proteoglycans present in these fractions. In $4 \mathrm{M}$ guanidine hydrochloride all proteoglycans were included on the gel, and no significant differences between the $\mathrm{CsCl}$ subfractions could be detected.

\section{Discussion}

A procedure has been developed for the preparation of proteoglycans which takes advantage of the situation prevailing when a cell culture and non-solid tissue is the source of the macromolecules. Thus separation of cell-associated and medium (matrix)derived proteoglycans is easily accomplished. Furthermore, proteoglycans from the cell media need not be subjected to any denaturing agents during the isolation procedure, which offers the possibility of isolating these macromolecules in a native state and of preserving, if present, any proteoglycan aggregates. In order to minimize the possible risk of proteolytic degradation by lysosomal proteinases from disintegrated cells, the various fractions were supplemented with proteinase inhibitors.

The preparation procedure described has been carried out five times on separate cell cultures of human embryonic skin fibroblasts with only minor differences in yield and in distribution of proteoglycans between the various fractions. Although a different isolation procedure was used, the total amount of heparan sulphate and dermatan sulphate in these fractions is in accordance with earlier work carried out at the glycosaminoglycan level (Sjöberg et al., 1979). 


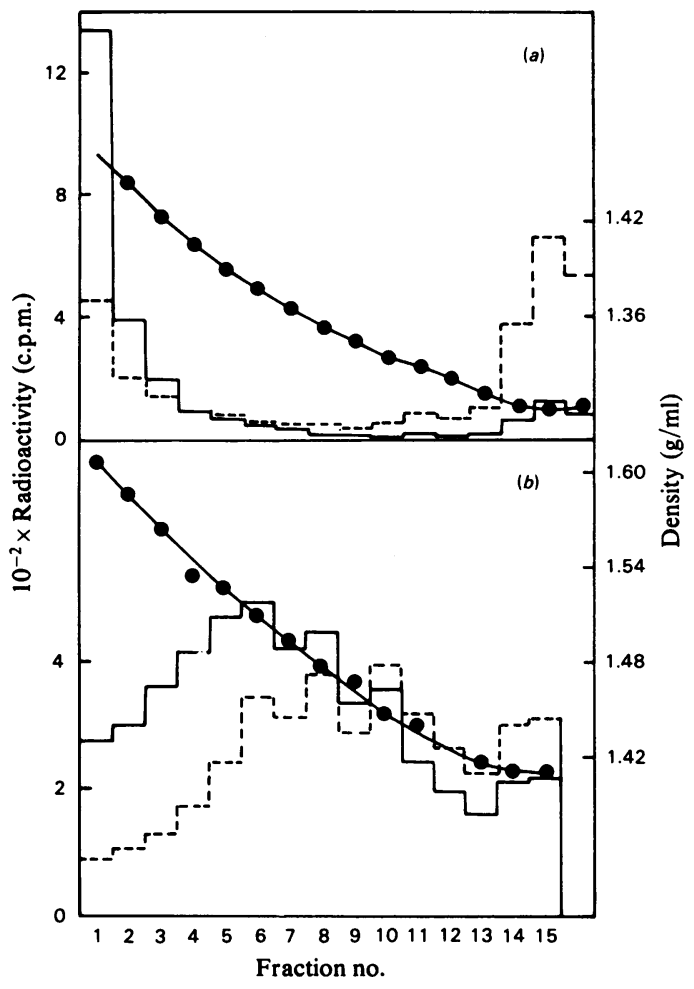

Gel chromatography of the extracts gave rise to subfractions of proteoglycans with different amounts of dermatan sulphate and heparan sulphate. Since dermatan sulphate isolated from cell cultures of human embryonic skin fibroblasts shows a considerable structural heterogeneity, it was of interest to assess the overall structure of the dermatan sulphate side chains derived from the different proteoglycan subfractions. This can be accomplished by controlled degradations followed by gel chromatography of the cleaved products. For this purpose, methods that selectively cleave non-sulphated iduronic acid residues (periodate oxidation followed by alkali treat-

Fig. 8. CsCl-density-gradient centrifugation of proteoglycans obtained from the medium

The proteoglycans (PG-M-I) were dialysed against $4 \mathrm{M}$-guanide hydrochloride/0.005 $\mathrm{M}$-Tris/ $\mathrm{HCl}, \mathrm{pH}$ 7.0.

(a) $\mathrm{CsCl}$ was added to a density of $1.35 \mathrm{~g} / \mathrm{ml}$. For further details see the Experimental section. Fractions 1-8 and 9-16 were pooled. (b) The pooled fractions 1-8 were subjected to a second density-gradient centrifugation. $\mathrm{CsCl}$ was added to this material until a starting density of $1.51 \mathrm{~g} / \mathrm{ml}$ was reached. Fractions 1-3, 4-6, 7-9, 10-12 and 13-15 were pooled. ${ }^{35} \mathrm{SO}_{4}$ (c.p.m.); ----, ${ }^{3} \mathrm{H}$ (c.p.m.); ๑, $\mathrm{CsCl}$ density.

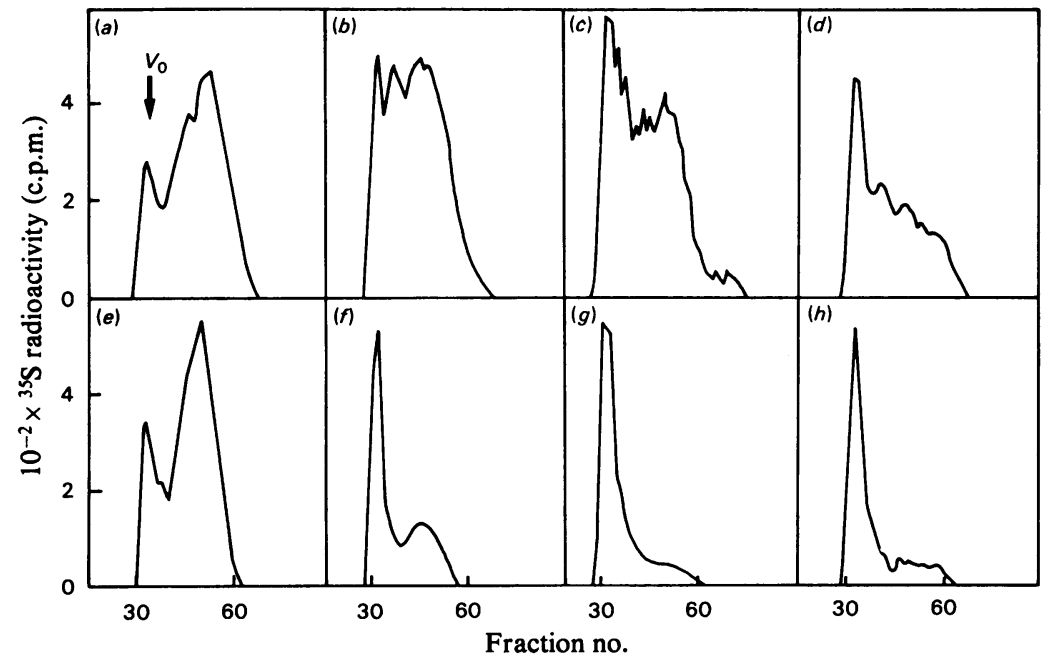

Fig. 9. Gel chromatography on Sepharose CL2B of subfractions of PG-M-I in the absence $(a-d)$ and in the presence $(e-h)$ of exogenous hyaluronic acid

Subfractions of PG-M-I with different buoyant densities, obtained by density-gradient centrifugation, were supplemented with $0.25 \mathrm{mg}$ of carrier A1D1D1-RA, dialysed against phosphate-buffereci saline and then chromatographed on a column of Sepharose CL2B $(a-d)$. Column size was $0.6 \mathrm{~cm} \times 140 \mathrm{~cm}$; elution rate was $3 \mathrm{ml} / \mathrm{h}$; fraction size was $0.6 \mathrm{ml}$; eluent was phosphate-buffered saline, pH 7.4. In one set of experiments $(e-h) 0.2 \mathrm{mg}$ of high-molecular-weight hyaluronic acid/ml was added to the proteoglycan subfractions, whereafter the samples were first dialysed against $4 \mathrm{M}$-guanidine hydrochloride/ $0.005 \mathrm{M}-\mathrm{Tris} / \mathrm{HCl}, \mathrm{pH} 7.0$, and then against phosphate-buffered saline before application to the column. $(a)$ and $(e)$ Fractions 1-3, $(b)$ and $(f)$ fractions 4-6, $(c)$ and $(g)$ fractions 7-9 and $(d)$ and $(h)$ fractions 10-12 in the absence and presence of hyaluronic acid respectively.

Vol. 183 
ment) and methods that cleave hexosaminidic linkages to glucuronic acid residues (chondroitinase AC digestion) were used, and dermatan sulphate was characterized as either iduronic acid-rich or glucuronic acid-rich (chondroitin sulphate-like). From the culture medium a proteoglycan subfraction (PG-M-I) devoid of heparan sulphate and characterized by unusually glucuronic acid-rich dermatan sulphate side chains was isolated. These dermatan sulphate species were only fragmented to a small extent by the periodate oxidation/alkaline elimination procedure (Fig. 3a). However, since most glycans changed their elution position after this treatment, it is concluded that small amounts of iduronic acid were present. By strict use of nomenclature, such glycans should be referred to as glucuronic acid-rich dermatan sulphatechondroitin sulphate co-polymers. The structural distinction from 'classical' chondroitin sulphate is negligible. It should be noted that free-radical-type depolymerization of glycosaminoglycan has been shown (Scott \& Tigwell, 1973). However, in the procedure used here the oxidation was carried out at $4^{\circ} \mathrm{C}$. Furthermore chondroitin sulphate subjected to the same treatment showed little depolymerization (Fransson, 1974). Finally, the result of the chondroitinase AC digestion show the presence of iduronic acid residues in tetra- and hexa-oligosaccharides, which suggests that L-iduronic acid-containing disaccharides are distributed mostly one or two together in the polysaccharide chains.

The material from the medium included on Sepharose 4B (fraction PG-M-II) contained both heparan sulphate and dermatan sulphate proteoglycans. The dermatan sulphate component of this proteoglycan fraction is characterized by a high content of iduronic acid (Fig. 3b).

The proteoglycans released from the cell layer by EDTA extraction closely resemble the ones derived from the culture medium. This similarity could be explained by physical entrapment of mediumdestined proteoglycans in the pericellular environment.

The reversible change in chromatographic behaviour noticed when fraction PG-M-I was chromatographed in guanidine hydrochloride and under physiological conditions could be attributed to either a dissociation-association phenomenon or to a conformational change. Since proteoglycans from the PG-M-I fraction have a much smaller Stokes radius in guanidine hydrochloride than in physiological saline, as shown by gel chromatography, we have favoured the hypothesis that this is due to dissociation of proteoglycan aggregates into smaller subunits rather than to a conformational change.

To rule out extrinsic (i.e. derived from the foetal calf serum used) or intrinsic (i.e. produced by the fibroblasts) proteins as the cause of this aggregation, the PGM-I fraction was further purified in a $\mathrm{CsCl}$ density gradient in guanidine hydrochloride. By this method a good separation is generally obtained between proteins (buoyant density lower than $1.35 \mathrm{~g} / \mathrm{ml}$ ) and glycoconjugates, which, depending on the ratio of carbohydrate/protein and on the number of charges, will equilibrate at densities between 1.35 and $1.70 \mathrm{~g} / \mathrm{ml}$. Since dermatan sulphate proteoglycans have a rather high protein content (Toole \& Lowther, 1968; Öbrink, 1972), ${ }^{35}$ S-labelled material with a buoyant density higher than $1.35 \mathrm{~g} / \mathrm{ml}$ was pooled. When these purified proteoglycans were chromatographed on Sepharose CL2B in guanidine hydrochloride and in physiological saline the same reversible association-dissociation phenomenon was observed. Non-proteoglycan proteins are therefore probably not the aggregating factors.

The second density gradient gave rise to subfractions of PG-M-I that also differed on gel chromatography. It is thus possible that fibroblasts synthesize more than one type of proteoglycan containing chondroitin sulphate-like side chains. Alternatively proteoglycans obtained at higher densities are species partially degraded by proteolytic enzymes. Such a proteolytic modification might abolish the ability of these proteoglycans to form aggregates.

The role of hyaluronic acid is unclear, and, although it promotes aggregation (Fig. 9), no proof of specificity has yet been obtained. However, hyaluronic acid is the principal glycosaminoglycan of the medium, and, since the binding site for hyaluronic acid on the carrier used (reduced and alkylated fraction A1D1D1) is destroyed, 'free' hyaluronic acid should be available in a large excess over fraction PG-M-I. Therefore endogenous hyaluronic acid could well be responsible for the aggregation observed before the addition of exogenous hyaluronic acid. The effect of the presence of carrier proteoglycan is difficult to assess, and the possibility of an interaction between A1D1D1-RA and fraction PG-M-I cannot be excluded under associative conditions. However, since the carrier is unable to interact with hyaluronic acid, the aggregation-promoting effect (see Fig. 9) exerted by this glycan cannot be due to the formation of mixed aggregates between A1D1D1-RA and fraction PG-M-I. It is therefore concluded that the phenomenon observed is due to an association between hyaluronic acid and fraction PG-M-I. This interpretation is also supported by the findings that hyaluronic acid promotes aggregation of similar proteoglycans from other mesenchymal tissues, such as glial cells (Norling et al., 1978) and aorta (McMurtrey et al., 1979; Oegema et al., 1979).

Alternatively the aggregation could be mediated by dermatan sulphate chain-chain interaction as described by Fransson (1976). However, binding to hyaluronic acid does not itself exclude the possibility of further aggregation due to dermatan sulphate chain-chain interaction. 
The PG-M-II fraction comprised $50 \%$ of the proteoglycans of the medium, and, when chromatographed in phosphate-buffered saline and guanidine hydrochloride, no difference in chromatographic behaviour was noticed. This observation further distinguishes the two dermatan sulphate proteoglycan species from the medium.

The origin of the dithiothreitol-extractable proteoglycans cannot be established at present, although they ought to be bound to a protein or proteoglycan structure on the cell membrane. An argument in favour of a localization at or close to the cell surface is the fact that cells do exclude Trypan Blue under the conditions used for the dithiothreitol extraction (Sjöberg et al., 1979). Proteoglycans containing iduronic acid-rich side chains were preferentially extracted by this procedure.

Proteoglycans released from the cells by guanidine hydrochloride (PG-C) should to a great extent be derived from the cell surface, as indicated by the high amount of heparan sulphate in this fraction (Kraemer, 1971). Also newly synthesized proteoglycans and proteoglycans undergoing degradation must be confined to this fraction, since virtually no proteoglycans are left in the extraction residue. The relative contribution of the possible sources to fraction PG-C is difficult to evaluate, but an approximate estimation can be achieved if the results of (Sjöberg et al. (1979) are considered. Trypsin was shown to release about $63 \%$ of the glycosaminoglycans left after EDTA extraction. Subsequent dithiothreitol extraction under conditions where the integrity of the plasma membrane was preserved (the cells excluded Trypan Blue) released about $7 \%$, and $4 \%$ of the glycosaminoglycans were found in a fraction assumed to represent intralysosomal material. Provided that cell-surface-associated material is completely removed by trypsin digestion, at most $11 \%$ of fraction PG-C could belong to a synthesis or a secretory pool. Like all other fractions, PG-C contains heparan sulphate proteoglycans and a mixture of iduronic acid-rich and glucuronic acidrich dermatan sulphate proteoglycans.

Thus a ubiquitous feature of all fractions seems to be the presence of at least two dermatan sulphate proteoglycan species characterized by glucuronic acid-rich and iduronic acid-rich glycosaminoglycan side chains. The relative proportion of these proteoglycan species and heparan sulphate proteoglycans varies from one fraction to the other. It is not known whether the iduronic acid-rich and glucuronic acidrich dermatan sulphate proteoglycans represent two discrete proteoglycans or represent two populations of interrelated species, nor could it be established at present whether the heparan sulphate proteoglycans from the various fractions are identical. Information on this must await studies directed towards the protein core of the proteoglycans.
This investigation was supported by grants from the Swedish Medical Research Council (B79-12X-139-13B), Greta och Johan Kocks Stiftelser, Trelleborg, Sweden, Gustaf V :s 80-års fond, Riksförbundet mot Reumatism and the Medical Faculty, University of Lund, Sweden. Valuable criticism by Dr. Lars-Åke Fransson and Dr. Ingrid Sjöberg and the expert technical assistance of Mrs. Lena Åberg and Miss Birgitta Jönsson is gratefully acknowledged.

\section{References}

Conrad, G. W., Hamilton, C. \& Haynes, E. (1977) J. Biol. Chem. 252, 6861-6870

Cöster, L., Carlstedt, I. \& Malmström, A. (1977) Upsala J. Med. Sci. 82, 125

Fransson, L.-A. (1974) Carbohydr. Res. 31, 339-348

Fransson, L.-Å. (1976) Biochim. Biophys. Acta 437, 106-115

Fransson, L.-Å. \& Carlstedt, I. (1974) Carbohydr. Res. 36, 349-358

Fratantoni, I. C., Hall, C. W. \& Neufeld, E. F. (1968) Proc. Natl. Acad. Sci. U.S.A. 60, 669-706

Grossfeld, A., Meyer, K., Godman, G. \& Linker, A. (1957) J. Biophys. Biochem. Cytol. 3, 391-396

Hardingham, T. E. \& Muir, H. (1974) Biochem. J. 139, 565-581

Hascall, V. C. \& Heinegård, D. (1974) J. Biol. Chem. 249, 4232-4241

Heinegård, D. (1972) Biochim. Biophys. Acta 285, 181-192

Heinegård, D. (1977) J. Biol. Chem. 252, 1980-1989

Kraemer, P. M. (1971) Biochemistry 10, 1445-1451

Kresse, H., v Figura, K., Buddecke, E. \& Fromme, H. G. (1975) Hoppe-Seyler's Z. Physiol. Chem. 356, 929-941

Lagunoff, R. \& Warren, G. (1962) Arch. Biochem. Biophys. 99, 336-400

Malmström, A., Carlstedt, I., Åberg, L. \& Fransson, L.-Å. (1975) Biochem. J. 151, 477-489

Matalon, R. \& Dorfman, A. (1966) Proc. Natl. Acad. Sci. U.S.A. 56, 1310-1316

McMurtrey, J., Radhakrishnamurthy, B., Dalferes, E. R., Berenson, G. S., \& Gregory, J. D. (1979) J. Biol. Chem. 254, 1621-1626

Norling, B., Glimelius, B., Westermark, B. \& Wastesson, A. (1978) Biochem. Biophys. Res. Commun. 84, 914-921

Öbrink, B. (1972) Biochim. Biophys. Acta 264, 354-361

Oegema, T. R., Jr., Hascall, V. C. \& Eisenstein, R. (1979) J. Biol. Chem. 254, 1312-1318

Saito, H. \& Uzman, B. G. (1971) Biochem. Biophys. Res. Commun. 43, 723-728

Scott, J. E. \& Tigwell, M. J. (1973) Carbohydr. Res. 28, 53-59

Sjöberg, I. \& Fransson, L.-Å. (1977) Biochem. J. 167, 383392

Sjöberg, I., Carlstedt, I., Cöster, L., Malmström, A. \& Fransson, L.-Å. (1979) Biochem. J. 178, 257-270

Suzuki, S., Saito, H., Yamagata, T., Anno, K., Seno, N., Kawai, Y. L. \& Furuhashi, T. (1968) J. Biol. Chem. 243, $1543-1550$

Toole, B. P. \& Lowther, D. A. (1968) Arch. Biochem. Biophys. 128, 567-578 